\title{
ZebraZoom: an automated program for high-throughput behavioral analysis and categorization
}

\author{
Olivier Mirat ${ }^{1,2}$, Jenna R. Sternberg ${ }^{1,3}$, Kristen E. Severi ${ }^{1}$ and Claire Wyart ${ }^{1 *}$ \\ Centre de Recherche de I'Institut du Cerveau et de la Moelle Épinière, UPMC, Inserm UMR S975, CNRS UMR 7225, Fondation ICM, Campus Hospitalier Pitié \\ Salpétrière, Paris, France \\ 2 Université Paris Descartes, Paris, France \\ ${ }^{3}$ Université Pierre et Marie Curie, Paris, France
}

\section{Edited by:}

Gonzalo G. De Polavieja, Instituto

Cajal. CSIC, Spain

\section{Reviewed by:}

Kristin Branson, HHMI Janelia Farm Research Campus, USA

Alfonso Perez-Escudero, Consejo

Superior de Investigaciones

Cientificas, Spain

*Correspondence:

Claire Wyart, Centre de Recherche de I'Institut du Cerveau et de la

Moelle Épinière, Inserm U975,

CNRS UPMC UMR 975, Fondation

ICM, Campus Hospitalier

Universitaire, Pitié-Salpétrière, 83,

Bld de I hôpital, 75013 Paris, France

e-mail: claire.wyart@

icm-institute.org
The zebrafish larva stands out as an emergent model organism for translational studies involving gene or drug screening thanks to its size, genetics, and permeability. At the larval stage, locomotion occurs in short episodes punctuated by periods of rest. Although phenotyping behavior is a key component of large-scale screens, it has not yet been automated in this model system. We developed ZebraZoom, a program to automatically track larvae and identify maneuvers for many animals performing discrete movements. Our program detects each episodic movement and extracts large-scale statistics on motor patterns to produce a quantification of the locomotor repertoire. We used ZebraZoom to identify motor defects induced by a glycinergic receptor antagonist. The analysis of the blind mutant atoh7 revealed small locomotor defects associated with the mutation. Using multiclass supervised machine learning, ZebraZoom categorized all episodes of movement for each larva into one of three possible maneuvers: slow forward swim, routine turn, and escape. ZebraZoom reached $91 \%$ accuracy for categorization of stereotypical maneuvers that four independent experimenters unanimously identified. For all maneuvers in the data set, ZebraZoom agreed with four experimenters in 73.2-82.5\% of cases. We modeled the series of maneuvers performed by larvae as Markov chains and observed that larvae often repeated the same maneuvers within a group. When analyzing subsequent maneuvers performed by different larvae, we found that larva-larva interactions occurred as series of escapes. Overall, ZebraZoom reached the level of precision found in manual analysis but accomplished tasks in a high-throughput format necessary for large screens.

Keywords: machine learning, tracking, analysis of kinematics, collective behavior, support vector machine classifier, multiclass categorization, locomotion in intact behaving animals
A central question in systems neuroscience is how neural circuit assembly and function relate to animal behavior. Genetic screens in invertebrate models, such as Drosophila melanogaster and Caenorhabditis elegans have begun to unravel the genetic basis of circuit function and behavior (Chalfie et al., 1985; Moore et al., 1998; Scholz et al., 2000). Automated methods have recently been developed in these species to track the position of individuals alone or in a group (Branson et al., 2009; Swierczek et al., 2011) and to categorize behavior (Dankert et al., 2009; Kabra et al., 2013). The zebrafish has emerged as an important vertebrate model organism for developmental biology, neurobiology, and human disease models, and is now used as a genetic model organism for the study of the mechanisms modulating complex behaviors in vertebrates such as depression and anxiety (Blaser et al., 2010; Lee et al., 2010; Cachat et al., 2011; Vermoesen et al., 2011; Zakhary et al., 2011; Ziv et al., 2013), sleep (Zhdanova et al., 2001; Appelbaum et al., 2009), or addiction (Petzold et al., 2009; Khor et al., 2011). The permeability, small size, genetic tractability, transparency, and low cost of zebrafish make them highly suitable for large-scale genetic and chemical screens (Driever et al., 1996; Granato et al., 1996; Haffter and Nusslein-Volhard, 1996).

Although simple for a vertebrate, the locomotor patterns of the zebrafish larva bring technical challenges to automated analysis. Larvae spontaneously swim in discrete bouts in a manner often described as "beat and glide," which can be classified as individual maneuvers, including slow forward swim, routine turn, or escape. These short movements are characterized by a large range of tailbeat frequencies $(15-100 \mathrm{~Hz})$, which require high-speed imaging to capture accurately and can be separated by long resting periods of up to a few seconds. Manual tracking via frame-by-frame analysis has formed the basis of contemporary knowledge and has enabled initial characterization of the larval zebrafish locomotor repertoire (Budick and O’Malley, 2000; Borla et al., 2002; McElligott and O'Malley, 2005). However, manual techniques are both laborious and limited in scope for high-throughput screens (Driever et al., 1996; Granato et al., 1996; Haffter and NussleinVolhard, 1996). The currently available automated tools have limitations in either refinement or time-scale. Recent chemical or genetic screens have relied on commercial software that estimates 
an index of mobility of the larvae, usually measured as the distance traveled during a recording session or the amount of time spent moving (Rihel et al., 2010; Elbaz et al., 2012; Rihel and Schier, 2012). These approaches for high-throughput screens provide information about average velocity and distance traveled by tracking the animals' center-of-mass over minutes to hours. Previous studies have either focused on analyzing movement duration and speed at low frequency over long periods of time or on fine analysis of kinematics at high frequency but for very short acquisition (typically $1000 \mathrm{~ms}$, Burgess and Granato, 2007; Liu et al., 2012). Accurate categorization of maneuvers for each individual in a group requires novel methods to record behavior with high temporal resolution and over long durations, automatically tracking and categorizing thousands of maneuvers.

Here we developed a new program, ZebraZoom, to track the full body position over a multiple-minute timescale of 56 larvae simultaneously recorded at high frequency and to finely characterize each maneuver. To identify core and tail positions for large datasets, videos were obtained on multiple larvae simultaneously over long periods of time and at high resolution using a highspeed camera run in a streaming-to-disk interface (Methods). Typically 500-1000 movements from seven larvae were recorded per dish in four minutes and eight dishes were monitored in parallel. To simplify tracking, we placed larvae in conditions that reduced overlapping in the z-plane during swimming (Methods; overlaps occurred on average once every $145 \mathrm{~s}$ per larva). We developed an offline 2D tracking method for identifying and separating each animal even when in close contact (Methods,
Figure 1). For each larva several features were identified, a core position that included the head and swim bladder (Figure 1A) and ten points along the tail (Methods and Figure 1B; Video S1). As movements occurred as discrete episodes, ZebraZoom detected movements based on the tail-bending angle over time (Methods and Figures 1C-D). To validate the accuracy of movement detection, one trained experimenter manually identified all movements occurring in a subset of videos. In three videos representing a total of 189 events, movements occurred with a false negative rate of $2.7 \%$ and a false positive rate of $3.7 \%$.

To quantify movements in a consistent manner, we used the location of the head, the position of the tail, the heading direction and the tail-bending angle to estimate global parameters of locomotion (Figure 2A, Methods). We observed that movements for 5-7 dpf wild-type (WT) larvae occurred every $2.22 \mathrm{~s}$ on average per larva (at $0.4495 \pm 0.0117 \mathrm{~Hz}$ ). For all movements identified, larvae performed on average $3.19 \pm 0.01$ oscillations per movement, had a $24.29 \pm 0.03 \mathrm{~Hz}$ tail-beat frequency (TBF), lasting $189.5 \pm 0.0004 \mathrm{~ms}$ with a $51.14 \pm 0.18^{\circ}$ heading direction range, $2.49 \pm 0.008 \mathrm{~mm}$ traveled distance and $13.35 \pm 0.04 \mathrm{~mm} / \mathrm{s}$ speed per maneuver. We illustrated the use of ZebraZoom for quantifying the effects of a known glycinergic receptor antagonist, and for analyzing a blind genetic mutant. Glycine is responsible for reciprocal inhibition in the spinal cord that permits left-right alternation to sustain oscillations (Dale, 1985; Grillner et al., 1995; Granato et al., 1996; Drapeau et al., 2002; Li et al., 2004). In zebrafish, mutants for glycinergic receptors or transporters have been associated with defects in motor pattern generation
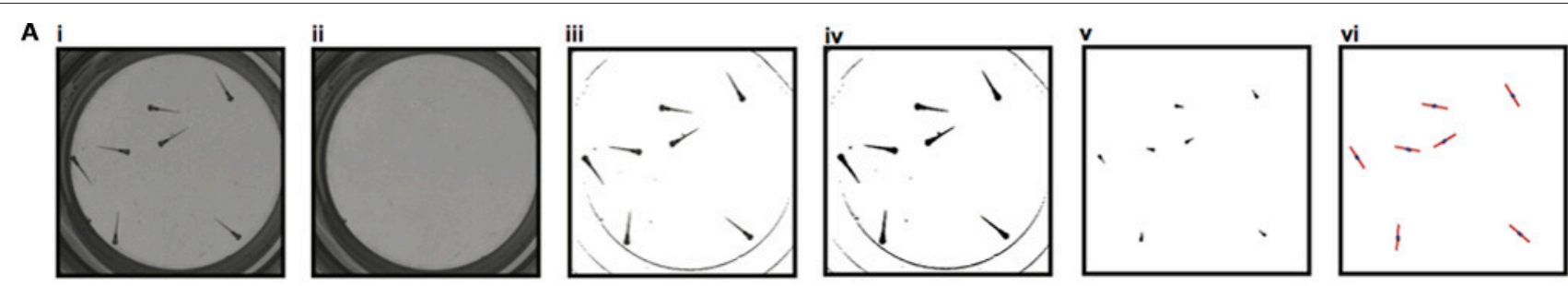

B i

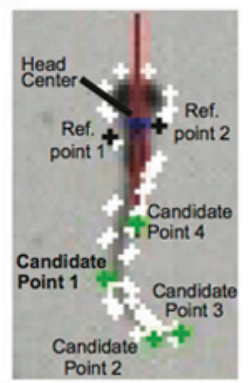

ii

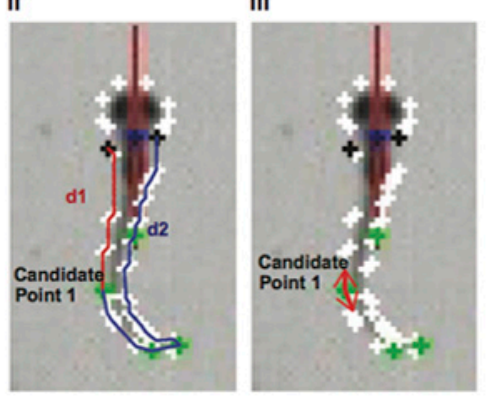

C

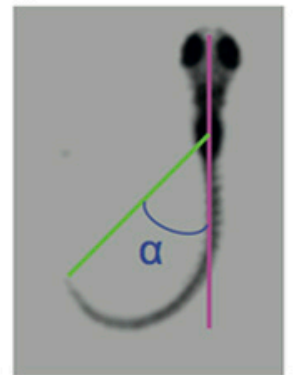

D

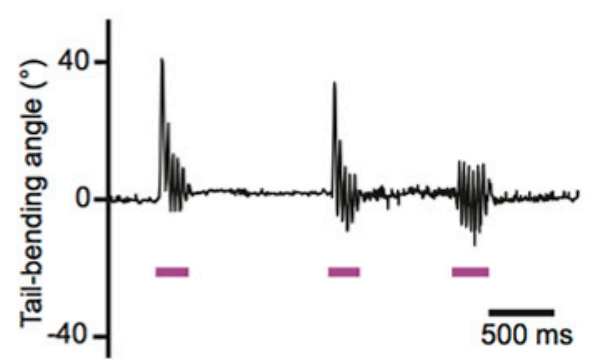

FIGURE 1 | Image processing for tracking of larvae's core positions and larvae's tail and detection of movements based on the tail-bending angle. (A) Tracking of the larvae's core positions. (Ai) Initial image. (Aii) Background image. (Aiii) Image with background subtracted. (Aiv) Binary image. (Av) Eroded image. (Avi) For each larva, identification of the core (blue dot) and heading direction (red axis). (B). Identifying the tip of the tail. (Bi) The head center is located at the boundary of the head and trunk. Candidate Point 1-4 along the tail are the four points of the contour with the smallest $x$-value, smallest $y$-value, largest $x$-value, and largest $y$-value caudal to reference points 1 and 2. (Bii) The two distances $\mathrm{d} 1$ and $\mathrm{d} 2$ shown for candidate point 1. (Biii) The two vectors used to identify the tail tip defined with the minimal scalar product for candidate point 1. (C) Definition of the tail-bending angle $(\alpha)$ separating the body axis (pink) and the line connecting the core and the tip of the tail (green).

(D) Example of the tail-bending angle over time with detection of movements indicated by the pink line. 


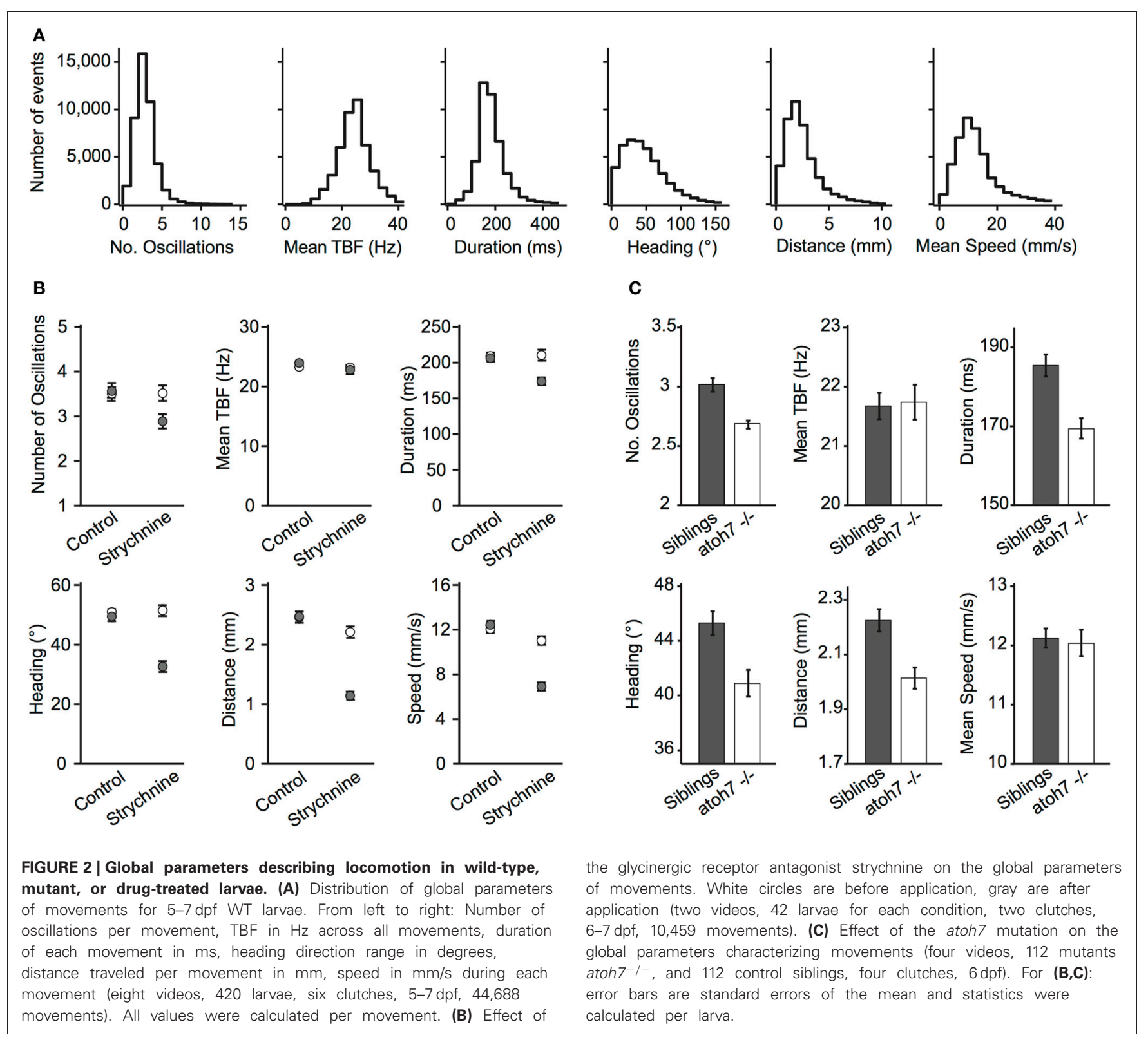

(Granato et al., 1996; Odenthal et al., 1996; Hirata et al., 2005; Masino and Fetcho, 2005). We measured the effect of bath application of $75 \mu \mathrm{M}$ strychnine on spontaneous locomotor activity in larvae and compared to control siblings that were not exposed to the drug (Figure 2B, Methods). For control larvae, we did not observe a significant change in the occurrence of movements over time $(0.35 \pm 0.05$ movements per larva/s before and $0.27 \pm 0.03$ movements per larva/s after), or on any of the global parameters (Figure 2B; all $p>0.15$ ). However the locomotor behavior of larvae treated with strychnine was significantly impacted (Figure 2B). Overall, movements occurred less frequently $(0.30$ $\pm 0.04 \mathrm{~Hz}$ before and $0.12 \pm 0.02 \mathrm{~Hz}$ after, $p<0.0002$ ). Although the average TBF during a movement did not change $(p>0.81)$, the number of oscillations decreased $(3.52 \pm 0.17$ before and 2.89 \pm 0.16 after; $p<0.0078)$, an effect that was associated with a decrease in movement duration $(p<0.0001)$, distance traveled $\left(p<10^{-5}\right)$, and average speed $\left(p<10^{-5}\right)$. Strychnine application also resulted in a decrease in the range of heading direction $\left(p<10^{-5}\right)$. atoh7 mutant larva lack retinal ganglion cells, rendering them blind (atoh $7^{-/-}$, Kay et al., 2001). Considering the importance of vision for zebrafish larvae, analyzing their locomotor output could reveal corresponding behavioral differences. Overall atoh $7^{-/-}$mutants generated episodic movements less frequently than control siblings $(0.33 \pm 0.02 \mathrm{~Hz}$ vs. $0.51 \pm$ $0.02 \mathrm{~Hz}, 112$ larvae for each condition). Quantitative analysis of global parameters of the blind mutants showed no difference in the average TBF or the average speed per larva (Figure 2C; $p>0.85$ and $p>0.83$, respectively) but there were small but significant decreases in the number of oscillations, duration, heading direction range, and distance traveled (Figure $2 \mathrm{C}$; all $p<10^{-3}$ ). 
These defects were observed systematically in four clutches. atoh $7^{-/-}$mutants thus display small but substantial differences in basic motor behavior when compared to control siblings.

Zebrafish larvae display a variety of locomotor maneuvers that are often grouped into discrete categories. In these experimental conditions, three types of movement occur in groups of larvae at early stages: slow forward swims $(\mathrm{S})$, routine turns ( $\mathrm{T}$, also referred to as slow turns), and escapes (E, including C-turns or burst swims). Figure 3 shows examples of these movements reported by ZebraZoom. For each maneuver, we superimposed a succession of images (Figures $\mathbf{3 A i}-\mathbf{C i}$ ), the tail-bending angle over time (Figures 3Aii-Cii) and the curvature along the rostrocaudal axis and as a function of time (Figures 3Aiii-Ciii). The three types of maneuvers included a series of slow left-right alternation; high values of curvature were confined to the caudal tail (Figures 3Aiii-Ciii). While high values of curvature of the tail were confined to the caudal end for slow forward swims (Figure 3Aiii), high values of curvature were distributed from head to tail for routine turns and escapes (Figures 3Biii,Ciii). Stereotypical routine turns and escapes differed by the frequency of left-right alternation in the tail bend (Figures 3Biii,Ciii). As larvae did not always exhibit a canonical slow forward swim, routine turn or escape, some movements were ambiguous. To estimate the percentage of these movements, four experimenters subjectively classified 390 movements distributed over eight videos. Overall about $82 \%$ of all movements were classified uniformly by at least three out of four experimenters (Methods) indicating that $18 \%$ of movements were difficult to categorize.

Using knowledge of stereotypical locomotor events, we designed a multiclass categorization approach with supervised machine learning to automatically sort each movement into one of the three categories. To implement the multiclass categorization, we used two successive support vector machine (SVM) classifiers: the first classifier sorted S vs. all other maneuvers, and when necessary the second classifier sorted T vs. E. Locomotor events were segregated subjectively in the training set $(n=201)$. This machine learning approach relied on associating dynamic parameters extracted from the tail-bending angle over time with each maneuver type identified in the training set (Figure 4A and Methods). To reduce the dimensionality of the data, we

Table 1 | Estimation of ZebraZoom categorizing accuracy based on the different reference experimenters.

\begin{tabular}{lllll}
\hline & All movements (\%) & $\boldsymbol{S ~ ( \% ) ~}$ & $\boldsymbol{T}(\%)$ & $\boldsymbol{E}(\%)$ \\
\hline Experimenter $\# 1$ & 82.5 & 85 & 82 & 79 \\
Experimenter $\# 2$ & 73.2 & 85 & 60 & 53 \\
Experimenter $\#$ 3 & 74.3 & 73 & 80 & 58 \\
Experimenter $\# 4$ & 75.4 & 79 & 77 & 47
\end{tabular}

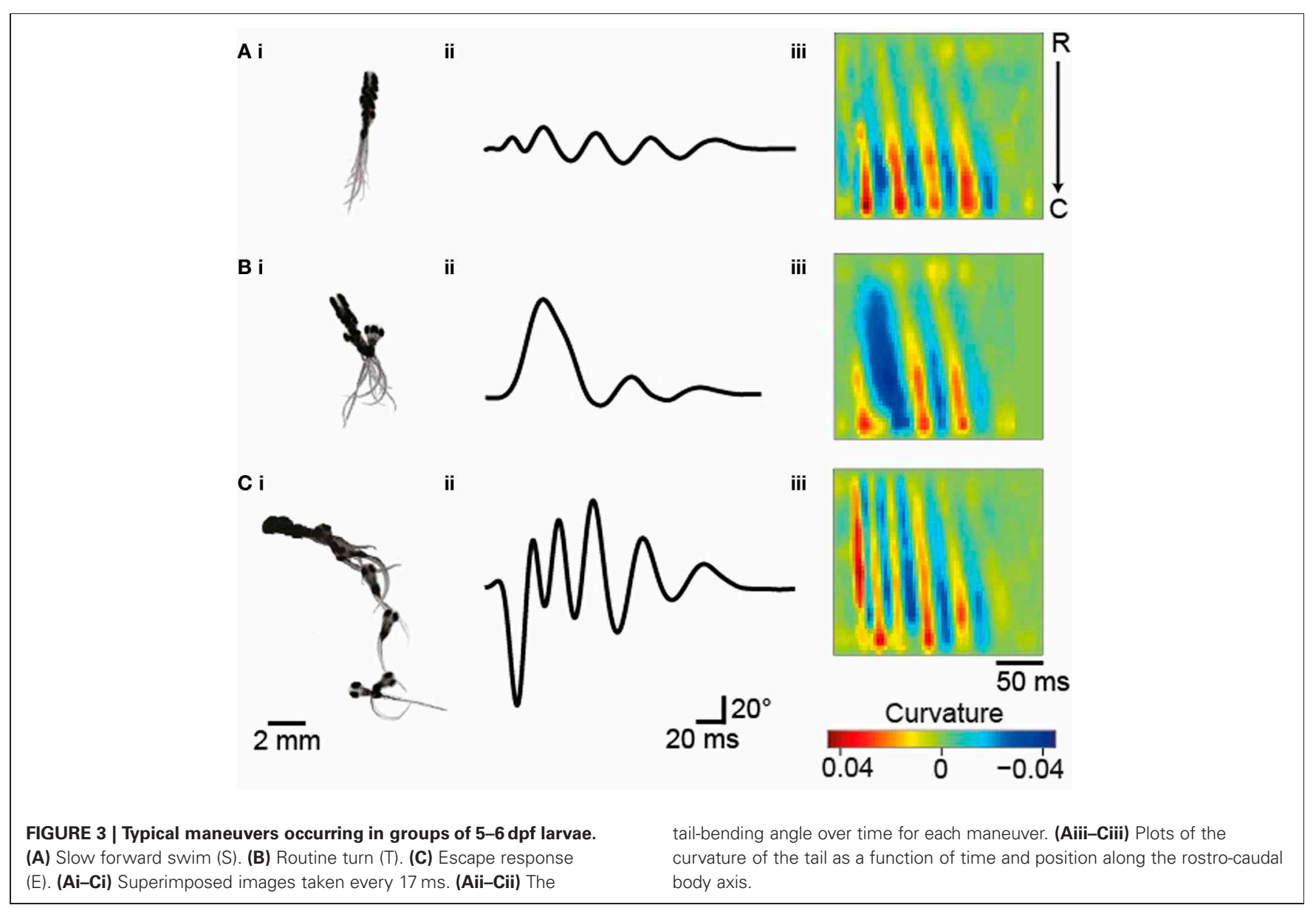


performed Principal Component Analysis (PCA). Based on the selection of a trained experimenter on the learning set, we validated the multiclass categorization to sort maneuvers by comparison with the subjective classification performed by a trained experimenter for a recognition set $(n=189$; Figures $4 \mathbf{4 B}, \mathbf{C})$. We observed that ZebraZoom agreed with the trained experimenter $82.5 \%$ of the time for the recognition dataset ( $85 \%$ for S, $82 \%$ for T, and 79\% for E; Figures 4B,C; Table $\mathbf{1}$ and Methods). When compared to four independent experimenters, ZebraZoom reached $91 \%$ accuracy for categorization of stereotypical maneuvers that all experimenters had unanimously identified and $76.4 \%$ on average for all maneuvers $(73.2-82.5 \%$, Table 1). Once validated, we applied the ZebraZoom categorization algorithm on a large dataset of 44,688 movements of WT larvae (Figure 4D). We identified 14.911 S (33.36\%), 21,432 T (49.96\%), and $8,345 \mathrm{E}(18.67 \%)$. The distribution of global parameters for the three classes of maneuvers were similar in terms of number of oscillations and duration, but they differed in terms of mean TBF, heading direction range, distance traveled and speed (Figure 4D).

The investigation of interactions between individuals leading to coordinated motion in animal groups has been a longstanding challenge that is central to elucidating the mechanisms and evolution of collective behavior. Most studies have focused on the analysis of speed or directionality to reflect the interaction between animals (Katz et al., 2011; Gautrais et al., 2012). We availed ourselves of ZebraZoom's features to accurately identify each larva and categorize their maneuvers to study how larvae interacted. In comparison to juvenile and adult zebrafish that swim continuously, larval zebrafish swim episodically with maneuvers that occur in a beat-and-glide manner. Each movement can be regarded as a discrete event, therefore we were motivated to explore how local perturbations of a single individual could impact the group. The program switched identity of larvae once every $109 \mathrm{~s}$ (once every 49 movements on average), allowing us to track single larvae. We modeled sequences of maneuvers performed by larvae within a group as Markov chains. Utilizing the classifier, we described larva-larva interactions in a group and intrinsic properties of individuals. We calculated a transition index $(I)$ for each sequence of two maneuvers as the transition probability between first and second maneuvers divided by the probability of random occurrence of the second maneuver (Figure 5; Table 2 and Methods). When the two successive maneuvers were the same, a higher transition index indicated the probability of repetition of this maneuver was greater than chance. The transition index was equal to one when the order of sequential maneuvers was random. Overall $I$ was greater than one for repetition of the same maneuvers (Table 2). We sorted the data into interactions between different animals and the repetition within the same animal. We analyzed how the transition index for a given succession of maneuvers depended on the distance between the two larvae's core positions at the onset of the movement and the time between the onset of each movement (Figure 5 and Methods). Individual larvae often performed the same type of maneuver sequentially (maximal values $I_{\mathrm{S} \max (\text { same) }}=1.43$, $I_{\mathrm{T} \max (\text { same })}=1.37, I_{\mathrm{E}}$ (same) $=2.38$, all $p<0.002$; Figure $5 \mathrm{~A}$,
Table 2, and Methods). Although slow forward swims or routine turns were not frequently repeated between larvae ( $I$ close to 1: maximal values $I_{\mathrm{S}}$ (diff) $=1.09$ and $I_{\mathrm{T}}$ (diff) $=1.01, p>$ 0.05 ; Figures 5Bi,ii, Table 2, and Methods), we found that recurrent escapes were very frequent between different larvae (maximal value $I_{\mathrm{E}}$ (diff) $=3.6, p<0.002$; Figure 5Biii, Table 2, and Methods). Five to seven dpf larvae do not show evidence for social interactions (Buske and Gerlai, 2011). By taking advantage of the algorithm for identifying single larva and categorizing simple maneuvers, we reveal that larva-larva interactions primarily occurred for escape responses. These series of escapes occurred after direct collisions (in one third of the cases) or via long distance interaction (two third of cases). Blind atoh $7^{-1-}$ larvae showed a similar profile of interactions for escapes (data not shown); these interactions were most likely mechanically triggered.

Large-scale chemical and genetic screens would benefit from a quantitative approach to analyze fine locomotor patterns over long periods of time. Compared to other genetic models, zebrafish locomotion is difficult to analyze because larvae initiate maneuvers intermittently and during these short events, the larvae swim at a high speed with TBFs ranging from $15-100 \mathrm{~Hz}$. The quantitative analysis of motor behavior for large-scale screens requires solving the problem of recording multiple animals simultaneously at high frequency (above $200 \mathrm{~Hz}$ ) and for long periods of time (minutes). Here we implemented a reliable method for quantifying global parameters of movements based on stream-todisk recordings acquired at high frequency and over long periods of time, limited only by data storage. Next we developed a robust method for tracking the full body position of zebrafish larvae swimming in groups. We first manually validated that the tracking accurately detected discrete movements, and then used the global parameters obtained to characterize the locomotion of WT larvae. Quantification of the global parameters describing larval movements corroborates previous observations based on fewer samples (Budick and O'Malley, 2000; Danos and Lauder, 2007; Liu et al., 2012). Similar estimates of the duration of movements, distance traveled and speed were obtained from the recent application of C-trax (designed originally for Drosophila) to zebrafish larvae [Lambert et al. (2012) based on Branson et al. (2009)]. In these conditions, recordings at low frequency over long periods of time, typically $60 \mathrm{~Hz}$ for minutes or hours, revealed the global level of activity over time but no information on fine kinematics during individual maneuvers (Elbaz et al., 2012). When recordings were performed at high frequency to capture the dynamics of motion, they usually lasted $1000 \mathrm{~ms}$ (Burgess and Granato, 2007).

We illustrated the benefit of ZebraZoom to quantify global parameters of movements by analyzing the effect of a drug to block glycinergic neurotransmission, which has been known to be involved in motor pattern generation and alternation between the left and right side of the spinal cord across vertebrate species (Grillner, 2003; Korn and Faber, 2005; Nishimaru and Kakizaki, 2009). Most studies relied on ventral nerve root recordings where muscles were dissected out or paralyzed in order to record the activity of motor neurons at the level of a few segments at most. Our automated quantification of locomotor events enabled 
A
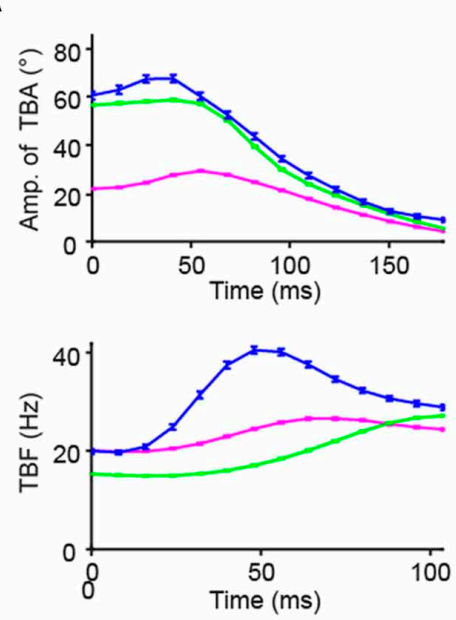

B
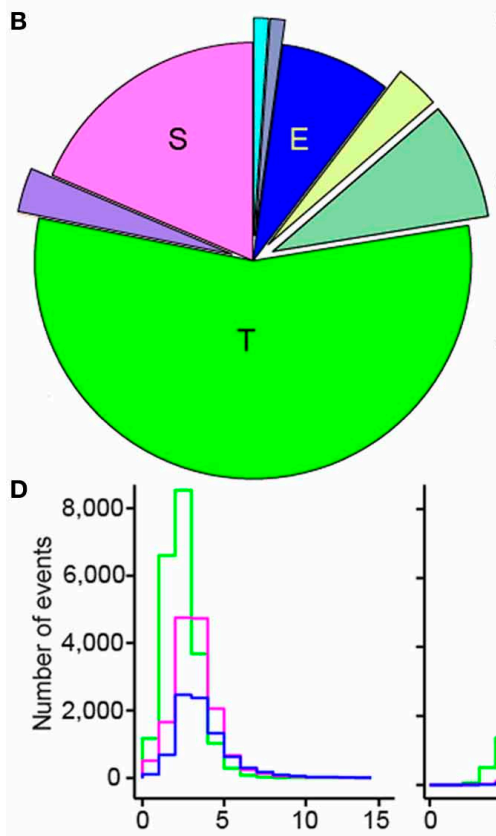

No. Oscillations

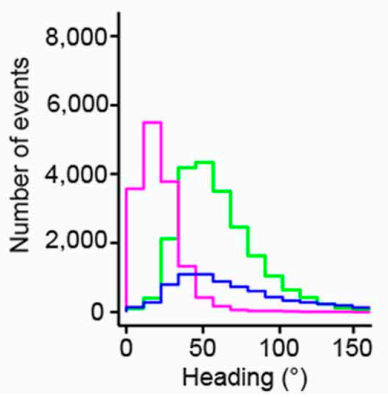

FIGURE 4 | Validation of the automated categorization of maneuvers: slow forward swim (S), routine turn (T) and escape (E). (A) Dynamic parameters used for categorizing the different maneuvers: amplitude of tail-bending angle (TBA) in degrees, integrated TBA in degrees, TBF in $\mathrm{Hz}$, and speed in $\mathrm{mm} / \mathrm{s}$. The mean of each parameter for each time bin is shown and error bars are standard error of the mean: $S$ in pink, $T$ in green and $E$ in blue. Time 0 is taken at the peak of the first bend of the movement. (B,C) Comparison of the results of the automatic categorization from
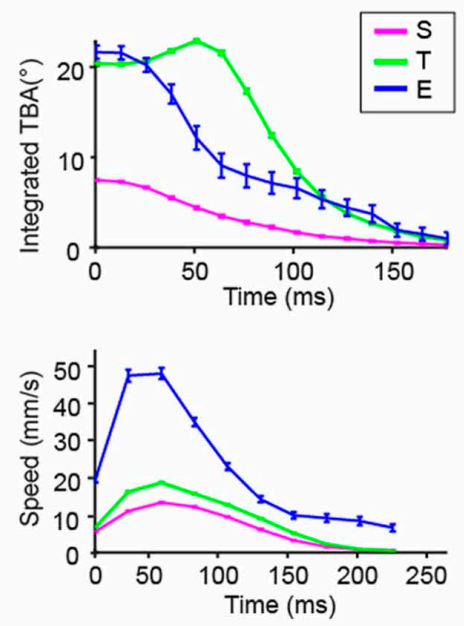

C i

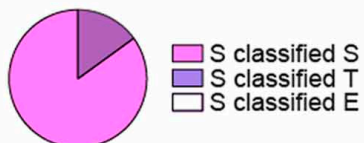

C ii

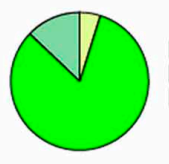

$\square$ T classified T

Tlassified S

T classified E

C iii

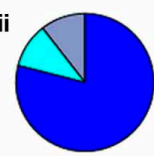

E classified E

Elassified $S$

$\square$ E classified T
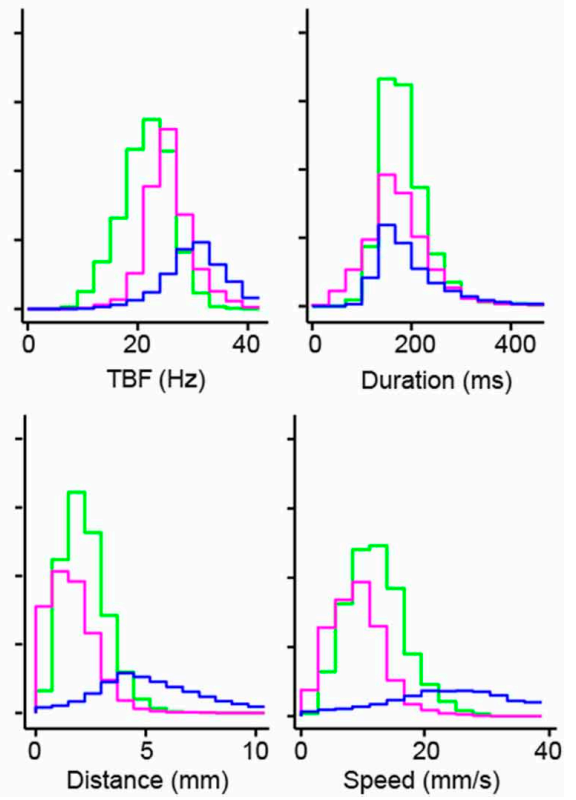

ZebraZoom with the subjective categorization by a trained experimenter on 189 movements from one video. The comparison of the categorization is shown overall (B) and for each maneuver (C: Ci for S, Cii for T, Ciii for E). The proportion of movements categorized the same way by both methods is shown in addition to the proportion of movements miscategorized and how they were categorized. (D) Distribution of global parameters for each maneuver S, T, and E of WT larvae (same color code as in $\mathbf{A} ; 44,688$ movements total from eight videos, six clutches). 

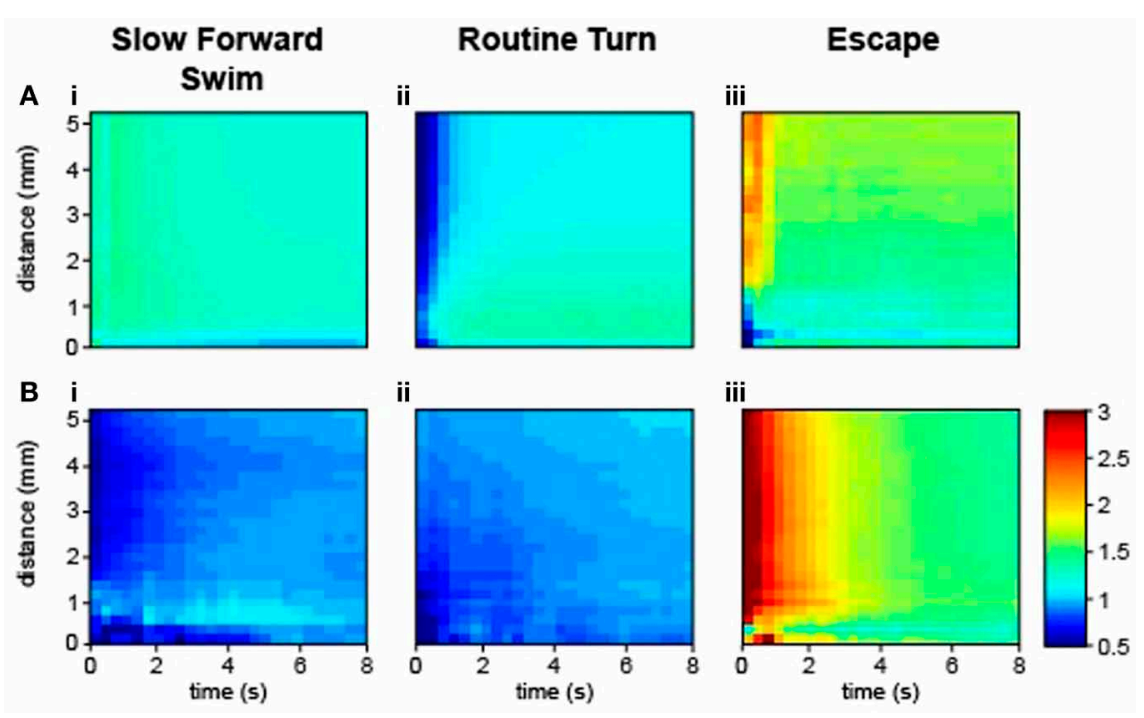

FIGURE 5 | Larva-larva interactions occurred most frequently as sequences of escapes. Transition index for the same larvae (A) and for different larvae (B). Time is plotted in seconds from the time of initiation of the first movement. Distance is plotted from the core position of the larva at the beginning of a movement. (Ai,Bi) The sequence S-S. (Aii,Bii) The sequence T-T. (Aiii,Biii) The sequence E-E (36,068 total movements from five videos, 280 WT larvae, four clutches, 5-6 dpf).
Table 2 | Transition index for sequence of two maneuvers estimated as the probability of transition from maneuver 1 to maneuver 2 divided by the occurrence of the maneuver 2 .

\begin{tabular}{lccc}
\hline & $\boldsymbol{S}$ & $\boldsymbol{T}$ & $\boldsymbol{E}$ \\
\hline \multicolumn{1}{l}{ INTERACTIONS } & BETWEEN DIFFERENT & ANIMALS \\
$\mathrm{S}$ & 1.0498 & 1.0152 & \\
$\mathrm{~T}$ & 1.0087 & 1.0413 & 0.8481 \\
$\mathrm{E}$ & 0.8549 & 0.8493 & 0.8606 \\
REPETITIONS WITHIN THE SAME ANIMAL & 1.7528 \\
S & 1.2162 & 0.8947 & \\
T & 0.9091 & 1.1185 & 0.8413 \\
E & 0.8263 & 0.8887 & 0.8499 \\
\hline
\end{tabular}

identification of effects induced by bath application of the glycinergic antagonist strychnine on locomotion in intact animals. As predicted, bath application of strychnine dramatically reduced the occurrence of movements and the number of oscillations per movement, that was correlated with a reduction of the duration of movement and of the distance traveled. While mean TBF was not affected, we observed a reduction in the heading direction range and in speed. Our approach pinpointed effects of glycinergic blockade, including a reduction in the number of oscillations per movement, a kinematic feature not estimated in commercially available software. The analysis of the mutant atoh7 revealed that although $\mathrm{TBF}$ and speed were not affected in the blind mutant, there was a small but significant decrease in the number of oscillations, heading direction range, distance, and duration of each bout compared to their control siblings. These effects were systematically observed on four clutches suggesting that visual feedback may impact some global parameters of locomotion. However since the pattern of expression of atoh 7 has not yet been fully characterized, it cannot be excluded that the gene may be expressed in cells other than retinal ganglion cells.

The originality of ZebraZoom lies in categorizing all maneuvers performed by individual larvae in a group. The subjective analysis of maneuvers based on four independent experimenters revealed that locomotor maneuvers were not obvious to categorize. Based on subjective estimates, $18 \%$ of all movements corresponded to ambiguous maneuvers. By using a machine-learning paradigm, we trained ZebraZoom to categorize all maneuvers over tens of thousands of movements with $82.5 \%$ accuracy, a similar value to the $72 \%$ agreement rate of all four experimenters measured over a few hundreds of movements. The approach we developed here could be expanded to include directionality of the turns, sequences of maneuvers such as those occurring during prey tracking, and subcategories of escapes.

This study constitutes an important first step for accurate tracking of multiple larvae in groups over long periods of time and for categorizing maneuvers. Some improvements could be implemented in the future. While our tracking method currently relies on a simple "blob" approach solely based on raw image analysis, a model-based approach may be more reliable in particular when animals are in close contact (Fontaine et al., 2008). We show here that ZebraZoom can achieve an accurate categorization of maneuvers, comparable to experimenters' estimates, based solely on the dynamics of movement of head and tail. An interesting avenue of exploration to address this could be investigation of novel dynamic parameters for the learning and recognition process of the classifier to yield subtler methods for detection of defects. Quantification of motor patterns in C. elegans is based on a description of all possible positions of the animal over time (Stephens et al., 2008). In order to fully understand larval zebrafish behavior we need to identify a minimal set of parameters sufficient to describe all motor patterns. All together this 
work brings new insight to the complexity of behavior determination in zebrafish larvae and could be applied to investigation of the mechanisms of addiction, arousal, feeding, social interaction and aggression in larvae and juveniles (Gahtan et al., 2005; Bianco et al., 2011; Buske and Gerlai, 2011; Miller and Gerlai, 2012; Ziv et al., 2013). The observation of complex interactions in juveniles raises the hope that it will soon be possible to investigate the neuronal circuits and molecular pathways underlying social interactions. The fact that we can track individual larva and analyze their interactions is a major advance over existing methods. Our approach that systematically quantifies and categorizes thousands of motor patterns was designed to bring efficiency and reliability to drug screening and forward genetic screens. ZebraZoom can detect, quantify, and categorize movements to provide a quantitative description of global parameters as well as a qualitative description of all maneuvers performed by individual larvae.

\section{METHODS}

\section{ZEBRAFISH HUSBANDRY}

All experiments were performed on Danio rerio larvae between 5 and $7 \mathrm{dpf}$. AB and TL strains of WT larvae were obtained from our laboratory stock of adults. Embryos and larvae were raised in an incubator at $28.5^{\circ} \mathrm{C}$ under a $14 / 10$ light/dark cycle (lights on, 8:00 A.M.; lights off, 10:00 P.M.) until the start of behavioral recordings. The mutant line for atoh7 (Kay et al., 2001) was given by Dr. Herwig Baier, MPI Munich. Double recessive atoh $7^{-/-}$ mutants were identified at $5 \mathrm{dpf}$ by their dark pigmentation. All procedures were approved by the Institutional Ethics Committee at the Research Center of the Institut du Cerveau et de la Moelle épinière (CRICM).

\section{BEHAVIORAL RECORDINGS}

Motor behavior of 56 larvae split into eight dishes (seven larvae per dish, Figure 1Ai) on a homogeneous illumination plate (light intensity $0.78 \mathrm{~mW} / \mathrm{cm}^{2}$, Phlox, ref. LEDW-BL-200/200-LLUB-Q-1R24) in egg water (http://zfin. org/zf_info/zfbook/chapt1/1.3.html, methylene blue added at $0.5 \mathrm{ppm}$ ). Following acclimation, larvae were recorded for $4 \mathrm{~min}$ at $337 \mathrm{~Hz}$ with a high-speed camera (VC-2MC-M340E0-C, CMOS chip $2048 \times 1088$ pixels, Vieworks, South Korea) placed above the setup and coupled to a camera objective (AF Nikkor $50 \mathrm{~mm}$ f/1.8D, Nikon, Japan). Pixel size was $66 \mu \mathrm{m}$. We developed a direct-to-disk high-speed imaging system designed for long acquisitions of raw images in collaboration with $\mathrm{R} \& \mathrm{D}$ Vision, France. Behavioral recordings were performed between 2:00 and 5:00 P.M. Larvae were acclimated for 60 minutes on the light source at room temperature $\left(21-22^{\circ} \mathrm{C}\right)$ and kept at room temperature during all recordings. Larvae were kept in dishes with an inner diameter of $2.2 \mathrm{~cm}$ and an outer diameter of $3.5 \mathrm{~cm}$ (Figure 1A). Water was kept at a low level $(2 \mathrm{~mm})$ in order to reduce the occurrence of crossings between larvae. Typically 500-1000 movements were recorded in each 4-min session for each well.

\section{ZEBRAZOOM TRACKING ALGORITHM}

The first step is to track the core and then the tail for all larvae over time. Written in $\mathrm{C}++$ using the open $C V$ library, the program identified the center position and heading direction of each larva (Figures 1Ai-vi). The algorithm used a Hough transform to identify the eight wells. For each well the background was estimated as the maximum pixel value over all frames of the video recording (Figure 1Aii) and then subtracted for all frames for that well (Figure 1Aiii). The resulting image was converted to binary (Figure 1Aiv). An erosion filter was applied twice in a row with a 3 by 3 structuring element (Figure 1Av). The "core" of the larva referred to the resulting connected components that had an appropriate area (between 0.0871 and $0.8712 \mathrm{~mm}^{2}$ ). The core of the larva included the head and the trunk with swim bladder (Figure 1Avi). The algorithm identified the head center position as the center of mass of the putative cores for each larva in a frame. To follow each larva across subsequent frames, ZebraZoom used the information from the previous two frames (core position and speed) to predict the position of the larva and located the closest core out of all the possible cores. The heading direction for each larva was calculated simultaneously using the moments of the eroded body (up to the second order, see red lines in Figure 1Avi).

For each larva with an identified core, we determined the "full body" referring to the connected component of the binary image in Figure 1Aiv. In order to track the tail, the full body was rotated so that the head axis was parallel to the y-axis, always in the same orientation. To identify the contour of the tail in the coordinate system defined by the head axis, a series of points was extracted from the full body by using the algorithm of Suzuki and Abe (1985), (white dots in Figures 1Bi-iii). Reference point A1 was the closest point on the contour line from the head center and reference point $\mathrm{A} 2$ was the point symmetrical along the head axis to reference point A1 on the contour. In order to identify the tip of the tail, four candidate points on the contour were selected with minimal and maximal $x$ - and $y$-values (Figures 1Bi-iii). For the maximal $y$-value the point also had to be above a given distance away from the two reference points [below a $20 \%$ threshold for the ratio $\left|\left(d_{1}-d_{2}\right)\right| /\left(d_{1}+d_{2}\right)$, Figure 1 Bii]. Distances $d 1$ and $d 2$ were calculated from each candidate point to the reference points A1 and A2 along the contour (Figure 1Bii). Candidate points with a ratio $\left|\left(d_{1}-d_{2}\right)\right| /\left(d_{1}+d_{2}\right)$ over 0.25 were excluded. The tip of the tail was then identified as the point associated with the smallest scalar product of the tangential vectors pointing in opposite directions (Figure 1Biii). The midline of the larva was defined as the line equidistant to the contour line on the left and right side.

\section{ERRORS IN CORE AND TAIL TRACKING}

If an error occurred in the core tracking, the larva was missing for that frame and there was no tracking of its tail. If the core of a larva was identified, the algorithm proceeded to the tail tracking. To confirm that the tail tracking was correct, the algorithm checked that the tail length was greater than 1.32 and less than $3.96 \mathrm{~mm}$. If this criterion was invalid, the tail position was set to the previous frame. This happened in $13.46 \%$ of frames on average but was compensated by a smoothing spline on the center positions between the left and right contour points of the tail and a median filter applied on the tail-bending angle over time. The tail-bending angle was defined as the angle between the axis formed by the tip of the tail and the center of the head with respect to the larva heading direction (Figures 1C,D). 


\section{SEPARATING LARVAE DURING CONTACTS}

Tracking was optimized to separate larvae in close vicinity to one another or in direct contact. For core tracking, if the trajectories of the two cores merged at a given time point, then the algorithm considered that a collision occurred between the two larvae. When the predicted positions of two larvae based on core position and speed in the two previous frames were closest to the same core, the algorithm considered that a collision between the two larvae occurred at that frame. When a collision was detected, the algorithm applied erosion filters in the region of interest defined by the core until more than one isolated core emerged. In rare cases, the multiple cores were not resolved and the larva could not be tracked for that frame. For the tail tracking, if the area of the larva's full body was greater than $1.9 \mathrm{~mm}^{2}$, the algorithm considered that two larvae were in direct contact. The distance separating the larvae's cores determined which of two algorithms was used to isolate the tails: if the distance was less than $1.32 \mathrm{~mm}$, a line separation algorithm was applied. A line was created to separate the two larvae by optimizing the area of the resulting tails, calculated by maximizing the sum of the two largest areas containing a head center position. If the distance was greater than $1.32 \mathrm{~mm}$, a pixel intensity separation algorithm was applied instead. The threshold used to convert the image into binary was adjusted until two separate full bodies, each a connected component, emerged and contained the head center position. Larvae crossings occurred once every $145 \mathrm{~s}$ on average per larva $(0.0069 \pm 0.0019$ events per second $)$ and the switching of identification between two larvae after a collision was estimated manually to occur every 109 s on average $(0.0092 \pm 0.0036$ events per second per larva based on $720 \mathrm{~s}$ of recordings from four videos, and 28 larvae).

\section{DETECTION OF MOVEMENTS}

Algorithms for the detection of movements and the behavior analysis were written in MATLAB (The Mathworks, Inc., USA). The detection of movement was based solely on thresholding the tail-bending angle measured over time (Figures 1C,D). ZebraZoom detected the start of a movement when the value for the tail-bending angle at a given frame varied over $1.15^{\circ}$ from the mean value of the tail-bending angle for the ten surrounding frames, or $29.7 \mathrm{~ms}$. To avoid separating single maneuvers into multiple events, movements that occur within $14.8 \mathrm{~ms}$ of each other were merged. To avoid false positives we considered only movements in which the larva core had moved more than $0.099 \mathrm{~mm}$ and where the range of tail-bending angle values was above 2.86 degrees. Additionally, only events during which the eroded binary image of the larva had moved more than a set number of pixels between subsequent images were considered based on the parameters used for the erosion. Rarely we have observed two distinct movements occurring without a pause, such as a slow forward swim followed by an escape due to a collision. In these few cases when two movements occurred without a noticeable stabilization in the tail-bending angle over time, the movements were merged into one movement in our analysis.

Our tracking method was robust in these experimental conditions. We cannot probe the impact of a reduction of contrast or spatial resolution. All numerical thresholds used above for tracking were fixed empirically, but they could easily be modified for other users to adapt to other recording conditions.

\section{CALCULATION OF THE CURVATURE}

After alignment of the body axis with the y-axis in a consistent orientation, the tail was represented parametrically in Cartesian coordinates as $[x(t), y(t)]$. The midline of the tail was fitted to the $x(t), y(t)$ function with a spline. Curvature was calculated in Cartesian coordinates:

$$
c=\frac{\left|x^{\prime} y^{\prime \prime}-y^{\prime} x^{\prime \prime}\right|}{\left(x^{\prime 2}+y^{\prime 2}\right)^{\left(\frac{3}{2}\right)}}
$$

where the derivatives were all calculated with respect to $t$, the distance along the tail.

\section{EXTRACTION OF GLOBAL PARAMETERS}

For all frames of a video, ZebraZoom outputs variables for each larva in each dish including: the position of its core, head axis, midline position of its tail and tail-bending angle. For each detected movement, a reference number for the larvae was extracted along with the corresponding well number, start and end time of the movement, and global parameters such as the number of oscillations, TBF, movement duration, heading direction range referring to the range of values of the heading axis for one movement with the heading angle reset to zero at the onset of movement, distance traveled, average speed (distance traveled divided by movement duration).

\section{AUTOMATIC MULTICLASS CATEGORIZATION}

We automatically attributed each movement detected in the video to either one of the three maneuvers: slow forward swim, routine turn or escape response. Our method relied on a dynamic set of parameters extracted from the bending angle of the tail estimated from the first tail bend over a limited time window (Figures 1C, 4A). We based our categorization on the four following parameters: (1) the amplitude of the tail-bending angle $(0-178 \mathrm{~ms}$, bins of $12 \mathrm{~ms})$, (2) the instantaneous frequency (0-104 ms, bins of $7 \mathrm{~ms}$ ), (3) the cumulative tail-bending angle calculated as the average angle value over time (0-178 ms, bins of $12 \mathrm{~ms})$, and (4) the speed (0-240 ms, bins of $24 \mathrm{~ms}$ ) (Figure 4A). The values of these four dynamic parameters were interpolated with a spline for a given time window during the movement and then used for categorization of every movement. PCA was first performed to reduce noise and dimensionality. Each movement was subsequently represented by the fourteen first principal components of the PCA out of 53 components (representing all together about $93 \%$ of the variance), to which the total duration of the movement was added. Multiclass categorization was implemented in two steps: a series of two subsequent SVM classifiers with linear kernel was applied for automatic categorization of movements: the first SVM classifier discriminated slow forward swims vs. turns and escapes, and if necessary a second SVM discriminated between a routine turn and an escape. We used two distinct datasets from WT 5-7 dpf larvae, one for learning the three maneuver types (five videos, $n=201$ movements) and one for testing their recognition (three videos, $n=189$ movements). 


\section{ESTIMATING THE RECURRENCE OF MANEUVERS}

Successions of maneuvers performed by larvae in a given dish were modeled as Markov chains. Out of the nine possible sequences of two maneuvers (S-S, S-T, S-E, T-S, T-T, T-E, E-S, E-T, E-E), we estimated the frequency of occurrence of each sequence. For a given movement classified as S, T, or E occurring at a given time in one dish, we calculated the transition probability for the subsequent movement to be classified as S, T, or E. We calculated a weighted transition index $(I)$ for each sequence of two sequential maneuvers as the ratio of the transition probability from the first maneuver to the second, divided by the probability of occurrence of the second maneuver (Table 2 for values of all transition indexes). When $I$ is equal to 1 , the probability of repeating a maneuver is equal to the probability of random occurrence of the maneuver (probability of random occurrence was 0.35 for S; 0.48 for $\mathrm{T}$ and 0.16 for E; Table 2 ). Thus the index of recurrence I was defined as:

$$
I(B 1, B 2)=\frac{p\left(x_{i}=B 1 \mid x_{i-1}=B 2\right)}{p\left(x_{i}=B 1\right)}
$$

with $B 1$ and $B 2$ as two possible maneuvers (S, T, or E) and $x_{i-1}$ and $x_{i}$ as two successive movements. WT larvae were used to estimate the transition index $(36,068$ movements from 280 larvae originating from four clutches and obtained from 40 wells). To investigate the recurrence of maneuvers as a function of time and distance, we calculated $I$ as a function of the distance separating the two head centers of the larvae at the onset of their respective movement and the time as the time interval between the onsets of the first and second movement. $I$ was calculated for many different time and distance windows. In Figure 5, we plotted these indexes for the sequences S-S, T-T, and E-E. We first calculated the index for the same larva (Figures 5Ai-iii) and across different larvae (Figures 5Bi-iii).

\section{STATISTICAL ANALYSIS}

The data used for Figure 2A were based on eight videos, $420 \mathrm{WT}$ $\mathrm{AB}$ larvae from six different clutches between 5 and $7 \mathrm{dpf}$. All values were given as mean \pm standard error of the mean (s.e.m.) calculated per movement. For the pharmacology experiments (Figure 2B), strychnine was bath applied at $75 \mu \mathrm{M}$ and the data were based on two videos of $84 \mathrm{WT}$ larvae coming from two clutches (42 for controls and 42 for strychnine) between 6 and $7 \mathrm{dpf}$. The data on atoh $7^{-/-}$mutants in Figure 2C were generated using four videos, 224 larvae total originating from four clutches (112 atoh $7^{-/-}$and 112 control siblings). All global parameters plotted in Figures 2B,C were calculated per larva then averaged across all larvae and means were given \pm s.e.m. across all larvae. Since the distributions of global parameters were not normal, a standard non-parametric Wilcoxon rank sum test was used in MATLAB for calculating differences between conditions with vs. without drugs for Figure $2 \mathbf{B}$ and $a t o h 7^{-/-}$vs. siblings for Figure 2C. The data used for Figure 4D were based on 44,688 movements from eight videos, 448 larvae, six clutches and for Figure 5 from 36,068 movements from five videos, $280 \mathrm{WT}$ AB larvae from four clutches. To test how the maximal values of the transition index were different from random, we calculated
$I_{\max }$ after randomly permuting maneuvers while keeping track of the larva identity, time, and location the same for 50 iterations. For each comparison, S-S, T-T, E-E across different larvae or within the same larva, we compared the values of $I_{\max }$ after randomization to the measured value $I_{\max }$ using a two-sample T-test.

\section{DATA AND ALGORITHM SHARING}

The software ZebraZoom is documented and available online from Source Forge in the code tab (http://sourceforge.net/p/ zebrazoom/wiki/Home/). ZebraZoom requires MATLAB and works reliably on an Ubuntu 11.04 computer with OpenCV installed and MATLAB 7.10.

\section{CONTRIBUTIONS}

Claire Wyart and Olivier Mirat designed the project with help from Jenna R. Sternberg. Olivier Mirat wrote all algorithms of the ZebraZoom program. Jenna R. Sternberg and Claire Wyart assembled the experimental setup. Jenna R. Sternberg performed all experiments. Olivier Mirat and Claire Wyart analyzed the data. Kristen E. Severi, Olivier Mirat, and Claire Wyart prepared the figures. All authors tested and validated the program and wrote the paper.

\section{ACKNOWLEDGMENTS}

We thank Dr. Stanley Durleman, Dr. Lionel Moisan, Dr. Carlos Pantoja, and Claire Oldfield for critical reading of the manuscript. We thank Dr. Olivier Colliot, and Drs. Stanley Durleman and Lionel Moisan again for advice on machine learning, tracking, and Markov chain analysis. We thank Caroline Vidal, Philippe Domineaux, and Antoine Mongay for data server access. We thank Laura Garnero, Audrey Baradel, Lydia Djenoune, Victor Adenis, Devika Nair, and Maxime Gaudin for technical assistance and fish care. We thank Dr. Herwig Baier for kindly sharing the atoh7 mutant line. This work received critical support from the Institut du Cerveau et de la Moelle Epinière (ICM/CR), the Ecole des Neurosciences de Paris (ENP), the Fondation Bettencourt Schueller, Mr. Pierre Belle, the City of Paris Emergence program, the ATIP-AVENIR program from INSERM and CNRS, the Fyssen Foundation, the International Reintegration Grant from Marie Curie Actions Framework Program 6, and the European Research Council (ERC) starter grant "OptoLoco." Jenna R. Sternberg is affiliated with the graduate program of the Ecole des Neurosciences de Paris (ENP) associated with the ED3C. Olivier Mirat is funded by a DIGITEO PhD fellowship associated with the Ecole Doctorale Interdisciplinaire Européenne Frontières du vivant ED474 program doctoral Bettencourt.

\section{SUPPLEMENTARY MATERIAL}

The Supplementary Material for this article can be found online at: http://www.frontiersin.org/Neural_Circuits/10.3389/ fncir.2013.00107/abstract

Video S1 | ZebraZoom tracking of seven larvae in a dish. Acquisition was performed at $300 \mathrm{~Hz}$, and one out of every ten images is displayed (every $33.3 \mathrm{~ms}$ ). 


\section{REFERENCES}

Appelbaum, L., Wang, G. X., Maro, G. S., Mori, R., Tovin, A., Marin, W., et al. (2009). Sleep-wake regulation and hypocretin-melatonin interaction in zebrafish. Proc. Natl. Acad. Sci. U.S.A. 106, 21942-21947. doi: 10.1073/pnas.906637106

Bianco, I. H., Kampff, A. R., and Engert, F. (2011). Prey capture behavior evoked by simple visual stimuli in larval zebrafish. Front. Syst. Neurosci. 5:101. doi: 10.3389/ fnsys.2011.00101

Blaser, R. E., Chadwick, L., and McGinnis, G. C. (2010). Behavioral measures of anxiety in zebrafish (Danio rerio). Behav. Brain Res. 208, 56-62. doi: 10.1016/ j.bbr.2009.11.009

Borla, M. A., Palecek, B., Budick, S., and O'Malley, D. M. (2002). Prey capture by larval zebrafish: evidence for fine axial motor control. Brain Behav. Evol. 60, 207-229. doi: 10.1159/000066699

Branson, K., Robie, A. A., Bender, J., Perona, P., and Dickinson, M. H. (2009). High-throughput ethomics in large groups of Drosophila. Nat. Methods 6, 451-457. doi: 10.1038/ nmeth. 1328

Budick, S. A., and O'Malley, D. M. (2000). Locomotor repertoire of the larval zebrafish: swimming, turning and prey capture. J. Exp. Biol. 203(Pt 17), 2565-2579.

Burgess, H. A., and Granato, M. (2007). Modulation of locomotor activity in larval zebrafish during light adaptation. J. Exp. Biol. 210( $\mathrm{Pt} \mathrm{14}$ ), 2526-2539. doi: 10.1242/jeb.003939

Buske, C., and Gerlai, R. (2011). Shoaling develops with age in Zebrafish (Danio rerio). Prog. Neuropsychopharmacol. Biol. Psychiatry 35, 409-1415. doi: 10.1016/j.pnpbp.2010.09.003

Cachat, J., Stewart, A., Utterback, E., Hart, P., Gaikwad, S., Wong, K., et al. (2011). Threedimensional neurophenotyping of adult zebrafish behavior. PLoS ONE 6:e17597. doi: 10.1371/ journal.pone.0017597

Chalfie, M., Sulston, J. E., White, J. G., Southgate, E., Thomson, J. N., and Brenner, S. (1985). The neural circuit for touch sensitivity in Caenorhabditis elegans. J. Neurosci. 5, 956-964.

Dale, N. (1985). Reciprocal inhibitory interneurones in the Xenopus embryo spinal cord. J. Physiol. 363, 61-70.

Dankert, H., Wang, L., Hoopfer, E. D., Anderson, D. J., and Perona, P. (2009). Automated monitoring and analysis of social behavior in Drosophila. Nat. Methods 6, 297-303. doi: 10.1038/nmeth.1310

Danos, N., and Lauder, G. V. (2007). The ontogeny of fin function during routine turns in zebrafish Danio rerio. J. Exp. Biol. 210( $\mathrm{Pt} 19)$, 3374-3386. doi: 10.1242/jeb.007484

Drapeau, P., Saint-Amant, L., Buss, R. R., Chong, M., McDearmid, J. R., and Brustein, E. (2002). Development of the locomotor network in zebrafish. Prog. Neurobiol. 68, 85-111. doi: 10.1016/S03010082(02)00075-8

Driever, W., Solnica-Krezel, L., Schier, A. F., Neuhauss, S. C., Malicki, J., Stemple, D. L., et al. (1996). A genetic screen for mutations affecting embryogenesis in zebrafish. Development 123, 37-46.

Elbaz, I., Yelin-Bekerman, L., Nicenboim, J., Vatine, G., and Appelbaum, L. (2012). Genetic ablation of hypocretin neurons alters behavioral state transitions in zebrafish. J. Neurosci. 32, 12961-12972. doi: 10.1523/JNEUROSCI.1284-12.2012

Fontaine, E., Lentink, D., Kranenbarg, S., Müller, U. K., van Leeuwen, J. L., Barr, A. H., et al. (2008). Automated visual tracking for studying the ontogeny of zebrafish swimming. J. Exp. Biol. 211(Pt 8), 1305-1316. doi: 10.1242/jeb.010272

Gahtan, E., Tanger, P., and Baier, H. (2005). Visual prey capture in larval zebrafish is controlled by identified reticulospinal neurons downstream of the tectum. J. Neurosci. 25, 9294-9303. doi: 10.1523/JNEUROSCI.2678-05.2005

Gautrais, J., Ginelli, F., Fournier, R., Blanco, S., Soria, M., Chaté H., et al. (2012). Deciphering interactions in moving animal groups. PLoS Comput. Biol. 8:e1002678. doi: 10.1371/journal.pcbi.1002678

Granato, M., van Eeden, F. J., Schach, U., Trowe, T., Brand, M., FurutaniSeiki, M., et al. (1996). Genes controlling and mediating locomotion behavior of the zebrafish embryo and larva. Development 123 , 399-413.

Grillner, S. (2003). The motor infrastructure: from ion channels to neuronal networks. Nat. Rev. Neurosci. 4, 573-586. doi: 10.1038/ nrn 1137

Grillner, S., Deliagina, T., Ekeberg, O., el Manira, A., Hill, R. H., Lansner, A., et al. (1995). Neural networks that co-ordinate locomotion and body orientation in lamprey. Trends Neurosci. 18, 270-279. doi: 10.1016/ 0166-2236(95)80008-P

Haffter, P., and Nusslein-Volhard, C. (1996). Large scale genetics in a small vertebrate, the zebrafish. Int. J. Dev. Biol. 40, 221-227.

Hirata, H., Saint-Amant, L., Downes, G. B., Cui, W. W., Zhou, W., Granato, M., et al. (2005). Zebrafish bandoneon mutants display behavioral defects due to a mutation in the glycine receptor betasubunit. Proc. Natl. Acad. Sci. U.S.A. 102, 8345-8350. doi: 10.1073/ pnas.0500862102

Kabra, M., Robie, A. A., Rivera-Alba, M., Branson, S., and Branson, K. (2013). JAABA: interactive machine learning for automatic annotation of animal behavior. Nat. Methods 10, 64-67. doi: 10.1038/nmeth.2281

Katz, Y., Tunstrøm, K., Ioannou, C. C., Huepe, C., and Couzin, I. D. (2011). Inferring the structure and dynamics of interactions in schooling fish. Proc. Natl. Acad. Sci. U.S.A. 108, 18720-18725. doi: 10.1073/pnas.1107583108

Kay, J. N., Finger-Baier, K. C., Roeser, T., Staub, W., and Baier, H. (2001). Retinal ganglion cell genesis requires lakritz, a Zebrafish atonal Homolog. Neuron 30, 725-736.

Khor, B. S., Jamil, M. F., Adenan, M. I., and Shu-Chien, A. C. (2011). Mitragynine attenuates withdrawal syndrome in morphine-withdrawn zebrafish. PLOS ONE 6:e28340. doi: 10.1371/journal.pone.0028340

Korn, H., and Faber, D. S. (2005). The Mauthner cell half a century later: a neurobiological model for decision-making? Neuron 47, 13-28. doi: 10.1016/j.neuron.2005. 05.019

Lambert, A. M., Bonkowsky, J. L., and Masino, M. A. (2012). The conserved dopaminergic diencephalospinal tract mediates vertebrate locomotor development in zebrafish larvae. J. Neurosci. 32, 13488-13500. doi: 10.1523/JNEUROSCI.1638-12.2012

Lee, A., Mathuru, A. S., Teh, C., Kibat, C., Korzh, V., Penney, T. B., et al. (2010). The habenula prevents helpless behavior in larval zebrafish. Curr. Biol. 20, 2211-2216. doi: 10.1016/j.cub.2010.11.025

Li, W. C., Higashijima, S., Parry, D. M., Roberts, A., and Soffe, S. R. (2004). Primitive roles for inhibitory interneurons in developing frog spinal cord. J. Neurosci. 24, 5840-5848. doi: 10.1523/JNEUROSCI.1633-04.2004

Liu, Y. C., Bailey, I., and Hale, M. E. (2012). Alternative startle motor patterns and behaviors in the larval zebrafish (Danio rerio). J. Comp. Physiol. A Neuroethol. Sens. Neural Behav. Physiol. 198, 11-24. doi: 10.1007/s00359-011-0682-1
Masino, M. A., and Fetcho, J. R. (2005). Fictive swimming motor patterns in wild type and mutant larval zebrafish. J. Neurophysiol. 93, 3177-3188. doi: 10.1152/ jn.01248.2004

McElligott, M. B., and O'Malley, D. M. (2005). Prey tracking by larval zebrafish: axial kinematics and visual control. Brain Behav. Evol. 66, 177-196. doi: 10.1159/ 000087158

Miller, N., and Gerlai, R. (2012). From schooling to shoaling: patterns of collective motion in zebrafish (Danio rerio). PLoS ONE 7:e48865. doi: 10.1371/journal.pone.0048865

Moore, M. S., DeZazzo, J., Luk, A. Y., Tully, T., Singh, C. M., and Heberlein, U. (1998). Ethanol intoxication in Drosophila: genetic and pharmacological evidence for regulation by the cAMP signaling pathway. Cell 93, 997-1007. doi: 10.1016/S0092-8674(00)81205-2

Nishimaru, H., and Kakizaki, M. (2009). The role of inhibitory neurotransmission in locomotor circuits of the developing mammalian spinal cord. Acta Physiol. (Oxf.) 197, 83-97. doi: 10.1111/ j.1748-1716.2009.02020.x

Odenthal, J., Rossnagel, K., Haffter, P., Kelsh, R. N., Vogelsang, E., Brand, M., et al. (1996). Mutations affecting xanthophore pigmentation in the zebrafish, Danio rerio. Development 123, 391-398.

Petzold, A. M., Balciunas, D. Sivasubbu, S., Clark, K. J., Bedell, V. M., Westcot, S. E., et al. (2009). Nicotine response genetics in the zebrafish. Proc. Natl. Acad. Sci. U.S.A. 106, 18662-18667. doi: 10.1073/pnas.0908247106

Rihel, J., Prober, D. A., Arvanites, A., Lam, K., Zimmerman, S., Jang, S., et al. (2010). Zebrafish behavioral profiling links drugs to biological targets and rest/wake regulation. Science 327, 348-351. doi: 10.1126/ science. 1183090

Rihel, J., and Schier, A. F. (2012). Behavioral screening for neuroactive drugs in zebrafish. Dev. Neurobiol. 72, 373-385. doi: 10.1002/dneu.20910

Scholz, H., Ramond, J., Singh, C. M., and Heberlein, U. (2000). Functional ethanol tolerance in Drosophila. Neuron 28, 261-271.

Stephens, G. J., Johnson-Kerner, B., Bialek, W., and Ryu, W. S. (2008). Dimensionality and dynamics in the behavior of C. elegans. PLoS Comput. Biol. 4:e1000028. doi: 10.1371/journal.pcbi.1000028

Suzuki, S., and Abe, K. (1985). Topological structural analysis of 
digital binary image by border following. Comput. Vis. Graph. Image Process. 30, 32-46.

Swierczek, N. A., Giles, A. C., Rankin, C. H., and Kerr, R. A. (2011). Highthroughput behavioral analysis in $C$. elegans. Nat. Methods 8, 592-598. doi: 10.1038/nmeth.1625

Vermoesen, K., Serruys, A. S., Loyens, E., Afrikanova, T., Massie, A., Schallier, A., et al. (2011). Assessment of the convulsant liability of antidepressants using zebrafish and mouse seizure models. Epilepsy Behav. 22, 450-460. doi: 10.1016/j.yebeh.2011.08.016
Zakhary, S. M., Ayubcha, D., Ansari, F., Kamran, K., Karim, M., Leheste, J. R., et al. (2011). A behavioral and molecular analysis of ketamine in zebrafish. Synapse 65, 160-167. doi: 10.1002/syn.20830

Zhdanova, I. V., Wang, S. Y., Leclair, O. U., and Danilova, N. P. (2001). Melatonin promotes sleep-like state in zebrafish. Brain Res. 903, 263-268. doi: 10.1016/S0006-8993 (01)02444-1

Ziv, L., Muto, A., Schoonheim, P. J., Meijsing, S. H., Strasser, D., Ingraham, H. A., et al. (2013). An affective disorder in zebrafish with mutation of the glucocorticoid receptor. Mol. Psychiatry 18, 681-691. doi: 10.1038/mp.2012.64

Conflict of Interest Statement: The authors declare that the research was conducted in the absence of any commercial or financial relationships that could be construed as a potential conflict of interest.

Received: 28 February 2013; accepted: 21 May 2013; published online: 12 June 2013.

Citation: Mirat $O$, Sternberg $J R$, Severi KE and Wyart C (2013)
ZebraZoom: an automated program for high-throughput behavioral anal$y$ sis and categorization. Front. Neural Circuits 7:107. doi: 10.3389/fncir. 2013.00107

Copyright (c) 2013 Mirat Sternberg, Severi and Wyart. This is an open-access article distributed under the terms of the Creative Commons Attribution License, which permits use, distribution and reproduction in other forums, provided the original authors and source are credited and subject to any copyright notices concerning any third-party graphics etc. 